\section{THE INDICATIONS AND UTILITY OF ELECTROENCEPHALOGRAM, AN AUDIT OF HOSPITAL PRACTICES}

Nicholas F Halliwell, Lucy Zhang, Stephan Golja, Ellie Skacel, Renee Pope, Callan A Coventry, Chinthuran Thilagarajan, Georgie CJ Dixson, Anna Schutz. Neurology, Gosford Hospital, Gosford, NSW, Australia

\subsection{6/bmjno-2021-ANZAN.48}

Objectives The MBS taskforce recommends discouraging use of electroencephalogram (EEG) investigations for low yield indications without neurological specialty input. This study aimed to examine the indications and utility of electroencephalogram within a hospital setting.

Methods A retrospective audit was undertaken over a 3 month period across two hospitals of adult inpatient EEGs ordered by services other than neurology.

Results Data was collected on 236 EEG encounters. 11\% of EEGs performed had a definitive diagnosis of seizures on discharge, of these EEGs the report documented; a normal EEG in $7 \%$, epileptiform activity in 19\%, and non-specific slowing in $74 \%$. $17 \%$ of Adult EEGs were performed for low yield indications. None of these EEGs resulted in change of management nor a diagnosis of seizures. An additional 14\% were performed as part of a 'falls work up,' none of the EEGs for this indication resulted in a diagnosis of seizure on the discharge summary.

Conclusion This audit supports previous findings that EEGs have a low sensitivity and can not be exclusively used to attain a diagnosis. Low yield indications were common within this audit and the EEG was not clinically significant in this group. EEG should not be used to rule out seizures when the clinical suspicion for seizures is near zero and this audit identified an additional low yield category within the hospital setting as part of a 'falls work up.' This study supports the conservative use of EEG in line with the MBS funding taskforce protocol.

\section{UNDER PRESSURE - AN UNUSUAL TRIGGER OF POSTERIOR REVERSIBLE ENCEPHALOPATHY}

James 0 Thomas, David B Williams, Christopher Grainge. John Hunter Hospital, New Lambton Heights, NSW, Australia

\subsection{6/bmjno-2021-ANZAN.49}

Background A 68-year-old female, and experienced recreational diver, presented via a regional hospital to our neurology service with acute onset visual disturbance following a 30metre open water dive.

She had no significant past medical history, specifically no history of hypertension and was on no regular medications. She was an ex-smother with a 20 pack year history

On arrival she was hypertensive to $190 / 100 \mathrm{mmHg}$ but otherwise systemically stable. She had intact visual acuity and visual fields but had difficulty distinguishing objects from complex backgrounds and described vivid visual distortions.

MRI performed acutely demonstrated multifocal areas of T2 hyperintensity within her posterior parietal and occipital lobes and a provisional diagnosis of posterior reversible encephalopathy syndrome (PRES) was made.

Further questioning revealed that the patient was diving with a 70/30\% nitrogen/oxygen mixture for which she had not received appropriate training, and was diving at depths close to the recommended limits for this mixture. Diving mixtures containing higher concentrations of oxygen are used to reduce the risk of nitrogen narcosis and decompression sickness but can be associated with CNS toxicity thought to be due to significantly increased $\mathrm{PaO} 2$ and associated cerebral vasoconstriction.

The patient was managed with intensive blood pressure control and made a significant recovery within seven days.

Conclusions We postulate that her PRES may have been triggered by excessive cerebral vasoconstriction from hyperoxaemia in a patient with impaired vasoregulatory reserve.

\section{REFERENCES}

1. Wingelaar TT, van Ooij P-JAM, van Hulst RA. Oxygen toxicity and special operations forces diving: hidden and dangerous. Front Psychol 2017;8:1263. doi:10.3389/fpsyg.2017.01263

2. Matsuo R, Kamouchi M, Arakawa S, Furuta Y, Kanazawa Y, Kitazono T. Magnetic resonance imaging in breath-hold divers with cerebral decompression sickness. Case Rep Neurol 2014;6(1):23-27. Published 2014 Jan 24. doi:10.1159/ 000357169

\section{PRESENCE OF VGCC ANTIBODIES WITH POSSIBLE LATE ONSET MULTIPLE ACYL-COA DEHYDROGENASE DEFICIENCY}

${ }^{1}$ Po Sheng Yang, ${ }^{1}$ Viral Upadhyay, ${ }^{2}$ Thomas Robertson, ${ }^{3}$ Robert Boots, ${ }^{1}$ Robert Henderson. ${ }^{1}$ Neurology, Royal Brisbane and Women's Hospital, Brisbane, QLD, Australia; ${ }^{2}$ Pathology of Queensland, Royal Brisbane and Women's Hospital, Brisbane, QLD, Australia; ${ }^{3}$ Thoracic Medicine, Royal Brisbane and Women's Hospital, Brisbane, QLD, Australia

\subsection{6/bmino-2021-ANZAN.50}

Introduction Voltage-gated calcium channel (VGCC) antibodies are considered specific for Lambert-Eaton myasthenic syndrome (LEMS). However, VGCC antibodies have been reported in other groups of patients without LEMS.

Multiple acyl-CoA dehydrogenase deficiency (MADD) is a type of mitochondrial myopathy which can present with a late onset form.

We report a case of a patient with positive VGCC antibodies concurrently with a diagnosis of possible late onset MADD.

Methods Clinical information and results of investigations were obtained.

Results A 75 year old woman presented with a gradual onset of proximal weakness associated with dyspnoea.

There was no relevant past medical history and family history was unremarkable.

Apart from mild proximal weakness, rest of the neurological examination was unremarkable. Deep tendon reflexes were normal.

Acylcarnitine profile on multiple occasions showed a pattern consistent with MADD. Muscle biopsy showed mild mitochondrial changes.

VGCC antibodies were detected on 2 separate occasions (86 and $100 \mathrm{pM;} \mathrm{Ref} \mathrm{Range}<30 \mathrm{pM}$ ). CMAP amplitudes and repetitive stimulation was normal with no facilitation nor decrement found. Position emission tomography was unremarkable.

The patient's symptoms were thought to be secondary to MADD, therefore was treated with riboflavin, Q10 and carnitine and described significantly improved proximal strength and function.

Conclusion It has been generally considered that the VGCC $\mathrm{Ab}$ has a high sensitivity and specificity for LEMS. However, 